\title{
Specialty Grand Challenge in Male Urology
}

\author{
Giorgio Ivan Russo * \\ Urology Section, Department of Surgery, University of Catania, Catania, Italy
}

Keywords: prostate, erectile and ejaculatory dysfunction, fertility, prostate cancer, andrology

\section{INTRODUCTION}

"Sexuality is a fundamental component of our being, even when we do not engage in any sexual activity (Anne Dickson)".

Yes, because sexuality today represents an increasingly consistent aspect of human life. With the increase in life expectancy and the prevention and treatment of significant pathologies of the organism, the sexual aspect represents a cornerstone of modern medicine. We must also not forget that unfortunately in the male sex, even today, the most frequent oncological diseases are those that affect the prostate gland, the bladder and even the kidney. For this reason, there is an increasing need for the urologist, but also other specialists to become real caregivers of the male patient.

In addition to the urologist's need to acquire more and more professionalism, there is also a need to ensure clinical responses to the patient's needs. According to our recent research carried out using the Google platform, we noticed that in the field of erectile dysfunction (ED), people increased their annual online research interest for prosthetic surgical treatment $(+1.7 \%)$, for drugs $(+0.7 \%)$, for mechanical treatment $(+0.6 \%)$ and for shockwave $(+1.8 \%)$ and on the contrary, we observed a reduction of interest in premature ejaculation decreased from 2004 to today (-1\%). These findings highlight the fact that patients are searching the web for sexual diseases treatment options and understanding their needs is much more important than the past (1).

Therefore, considering these premises, scientific research concerning male urology is facing an unprecedented boom.

Therefore, there is an increasing need to combine the highest scientific advances with the most recent clinical and therapeutic advances in order to keep up with the times and continue to look to the future.

giorgioivan.russo@unict.it

*Correspondence:
Giorgio Ivan Russo

Specialty section:

This article was submitted to

Male Urology,

a section of the journal

Frontiers in Urology

Received: 12 May 2021

Accepted: 04 June 2021

Published: 15 June 2021

Citation:

Russo Gl (2021) Specialty Grand

Challenge in Male Urology.

Front. Urol. 1:708869.

doi: 10.3389/fruro.2021.708869

\section{RESEARCH CHALLENGES}

When it comes to the treatment of lower urinary tract symptoms (LUTS), several pharmaceutical categories have long been used. However, even today we have not been able to take adequate action on prevention.

If we look deeper, and consider various risk factors more generally, certainly the role of obesity is playing a fundamental role. In particular, BPH tissues exhibited enrichment of myofibroblast subsets but also depletion of neuroendocrine cells and an estrogen receptor - positive fibroblast cell type residing near the epithelium.

Genetic study on whole-exome sequencing has demonstrated that genomic characterization of $\mathrm{BPH}$ has identified a clinically relevant stromal signature and new candidate disease pathways 
(including a likely role for BMP5 signaling) and reveals BPH to be not merely a hyperplasia, but rather a fundamental relandscaping of cell expansion (2).

We must therefore continue to look deeper and deeper and then look to the future through the implementation of gene treatments and understand how to act on prevention.

It is important to notice that the success of the metastatic process is conditioned by the established relationship between tumor cells and the surrounding microenvironment (3). During the metastatic process, tumor cells interact with the immune system, which modulates this process, by the formation of CTC clusters or microemboli also influenced by gut microbiome (4).

The gut microbiome contributes to disease progression through different mechanisms (5) and microbes can also alter cancer cell epigenetics through production of metabolites affecting gene expression (6). In fact, it has been recently demonstrated that the microbiome and related dysbiosis affect metabolic process in patients. This interestingly interplay between microbiome and gut can influence the development of MetS in patients with BPH due to the production of fatty acids. Authors also showed that the production of short chain fatty acids is accompanied by the production of harmful metabolites that contribute to the damage of the intestinal barrier and consequently to the penetration of bacterial and inflammatory factors into the blood with increasing of prostate gland inflammation and proliferation of prostate cells (7).

\section{PHARMACOLOGICAL CHALLENGES}

Of all the pathologies related to male urology, erectile dysfunction and benign prostatic hyperplasia are certainly the most common. Reptile dysfunction mainly affects patients over the age of forty and has a negative impact on sexual and relationship life as a couple. It has been estimated that by 2025 approximately 322 million males will suffer from erectile dysfunction with an increase of more than $100 \%$ compared to 1994 (8). Although notable leaps and bounds in the pharmacological field have been made since the 1990s, primarily with regard to phosphodiesterase type-5 inhibitors (PDE5is), no further steps have been taken since then. A look to the future, for example, may concern the use of stem cells. These are undifferentiated or partially differentiated cells, which are characterized by the possibility of self-renewal and above all differentiating into cells of different specialties. Their particular ability to divide and also in the regeneration of tissue organs is influenced by the environment, making them potentially suitable for the treatment of various diseases. In fact, their importance is expressed in a general application through an anti-inflammatory activity and through their ability as immunomodulators in the cardiovascular and other fields (9). Precisely for this reason stem cell therapy can be coupled with the treatment of erectile dysfunction thanks to their potential to transform into endothelial, neuronal or smooth muscle cells and also repair possible damage to the penile tissue. Current and near future research must certainly direct the treatment towards certainly regenerative approaches.

Several treatments have always been used for the treatment of LUTS secondary to benign prostatic hyperplasia. However, we are still a long way from being able to prevent it.

Many evidences have long shown that the metabolic syndrome (MetS) is associated with greater severity of symptoms and greater disease progression. Interestingly, these premises raised the question of the possible efficacy of statins through inhibition of 3-hydroxy-3-methylglutaryl-coenzyme A (HMG-CoA) and reductions in total cholesterol, low-density lipoprotein, apoprotein B and triglycerides (10).

In vitro studies have in fact shown how they are able to inhibit the proliferation of prostate cells.

Harshman et al. showed that statins inhibit cell androgen uptake by competing for intracellular transport sites in the solute carrier organic anion transporter family, member 2B1 (SLCO2B1). Specifically, the uptake of dehydroepiandrosterone sulphate (DHEAS), a precursor of potent androgens such as dihydroxytestosterone (DHT), is consistently reduced by statin exposure. Thus, statins may reduce intracellular androgen supply (11).

In addition to this translational efficacy, these drugs with long-term administration (at least 1 year) would appear to reduce the risk of disease progression.

Taking all these premises together, Oria is required to carefully evaluate the drugs that can act preventively on benign prostatic hyperplasia, acting at several levels also from a metabolic point of view, precisely to improve symptoms but at the same time also reduce progression. of the disease itself.

\section{SURGICAL CHALLENGES}

Considerable advances in the surgical field have been promoted in the surgical field and for congresses, especially as regards $3 \mathrm{D}$ printing. Although more advances have been made in the oncology field, we are still behind in some andrological fields.

3-dimensional printing has revolutionized healthcare in the last years, leading to the building of models and customized sample that helps physician and patients for a better understanding of the disease. One the most interesting application may include the creation of penile prosthesis that can be tailored on the patient and even to simulate pre- and perioperative surgical plans (12).

Behind this aspect, the revolutionary role of $3 \mathrm{D}$ printing has a role also during congresses. Interestingly, Amparore et al. have investigated this perception among attendees of the 8th TechnoUrology-Meeting 2021. Overall, augmented reality was the preferred option for intraoperative guidance and training, in both prostate (55\% and 38.3\%) and kidney cancer surgery $(58.3 \%$ and $40 \%$ ) while HoloLens was perceived as the best imaging technology for the surgical planning (50\% for prostate and $60 \%$ for kidney), whereas printed models for patients counselling (66.7\% for prostate and $61.7 \%$ for kidney) (13). 
To this regard, also a special attention should be given to the surgical approaches for LUTS/BPE that are increasing with time and the choice of the right technique for the right patient depends on several factors such as prostate size, comorbidities, surgeon experience, and willingness to accept surgery-associated side-effects (14).

In particular, despite the high efficacy of current surgical treatments, the rates of ejaculatory dysfunction $(\mathrm{EjD})$ remain the most important issue for patients.

For this reason, new treatments new treatment modalities may have an impact on anterograde ejaculation preservation after endoscopic surgery in patients with $\mathrm{BPH}$, without compromising efficacy when compared with standard procedure. The application of new laser-based or water-based technologies in order to preserve anatomical structures together with proper enucleation techniques improve ejaculatory dysfunction.

\section{PSYCHOLOGICAL PERSPECTIVE}

Apart from all the previous premises concerning male urology, we believe that a particular look must be taken with regard to the psychological aspects of sexual health, not necessarily identifiable with a gender. To date, gender identity and the science that concerns it must make an even greater effort to amalgamate the different specialties that are able to cooperate in order to give specific answers to all patients.

For example, let's look at the concept of asexuality that is increasingly playing a role in patient management. Asexuality is currently accepted by many researchers as a lifelong lack of sexual attraction and in detail, asexuals represents low rate (1\%) of the general population, but statistics are not certain on this aspect (15).

\section{REFERENCES}

1. Russo GI, di Mauro M, Cocci A, Cacciamani G, Cimino S, Serefoglu EC, et al. Consulting "Dr Google" for Sexual Dysfunction: A Contemporary Worldwide Trend Analysis. Int J Impot Res (2020) 32(4):455-61. doi: 10.1038/s41443019-0203-2

2. Middleton LW, Shen Z, Varma S, Pollack AS, Gong X, Zhu S, et al. Genomic Analysis of Benign Prostatic Hyperplasia Implicates Cellular Re-Landscaping in Disease Pathogenesis. JCI Insight (2019) 5. doi: 10.1172/jci.insight.129749

3. Garrido-Navas C, de Miguel-Perez D, Exposito-Hernandez J, Bayarri C, Amezcua V, Ortigosa A, et al. Cooperative and Escaping Mechanisms Between Circulating Tumor Cells and Blood Constituents. Cells (2019) 8 (11):1382. doi: $10.3390 /$ cells8111382

4. Li W, Deng Y, Chu Q, Zhang P. Gut Microbiome and Cancer Immunotherapy. Cancer Lett (2019) 447:41-7. doi: 10.1016/j.canlet.2019.01.015

5. Sieling PA, Chung W, Duong BT, Godowski PJ, Modlin RL. Toll-Like Receptor 2 Ligands as Adjuvants for Human Th1 Responses. J Immunol (2003) 170:194-200. doi: 10.4049/jimmunol.170.1.194

6. Gerhauser C. Impact of Dietary Gut Microbial Metabolites on the Epigenome. Philos Trans R Soc B Biol Sci (2018) 373:20170359. doi: 10.1098/ rstb.2017.0359

7. Ratajczak W, Mizerski A, Rył A, Słojewski M, Sipak O, Piasecka M, et al. Alterations in Fecal Short Chain Fatty Acids (Scfas) and Branched ShortChain Fatty Acids (Bcfas) in Men With Benign Prostatic Hyperplasia (BPH) and Metabolic Syndrome (Mets). Aging (Albany NY) (2021) 13(8):10934-54. doi: 10.18632/aging.202968
A very recent review on this topic suggested that asexuals present great heterogeneity of sexual behaviors and psychological processes regarding sexuality, including different aspects and types of interpersonal and romantic relationships, sexual attitudes or fantasies.

Moreover, special attention can be given to Lesbian, gay, bisexual, transgender, and queer (LGBTQ) since researches on this topic, regarding its impact in oncology, surgery and sexuality merits much more attention. In fact, we suggest that more support groups specifically for LGBTQ should be established, and urologists and other specialist should give to the sexual and implications of treatment, and not frame problems for LGBTQ in heterosexual terms.

\section{FINAL REMARKS}

Although it is not possible to offer a vision of the future regarding male urology, the journal Frontiers in Urology offers the maximum contribution in the field of research on many aspects of urology in order to improve knowledge in this area especially for our patients and for clinicians. In addition to the topics covered by this editorial, there are many other interesting arguments capable of covering scientific gaps still present today. It will in fact be our task to fill these gaps.

\section{AUTHOR CONTRIBUTIONS}

The author confirms being the sole contributor of this work and has approved it for publication.

8. Ayta IA, McKinlay JB, Krane RJ. The Likely Worldwide Increase in Erectile Dysfunction Between 1995 and 2025 and Some Possible Policy Consequences. BJU Int (1999) 84:50-6. doi: 10.1046/j.1464-410x.1999.00142.x

9. Chen S, Liu Z, Tian N, Zhang J, Yei F, Duan B, et al. Intracoronary Transplantation of Autologous Bone Marrow Mesenchymal Stem Cells for Ischemic Cardiomyopathy Due to Isolated Chronic Occluded Left Anterior Descending Artery. J Invasive Cardiol (2006) 18:552-6.

10. Russo GI, Larganà G, Sebastianelli A, Cocci A, Di Mauro M, Rapallo I, et al. The Investigative Role of Statins in Ameliorating Lower Urinary Tract Symptoms (LUTS): A Systematic Review. J Clin Med (2021) 10(3):416. doi: $10.3390 / \mathrm{jcm} 10030416$

11. Harshman LC, Wang X, Nakabayashi M, Xie W, Valenca L, Werner L, et al. Statin Use at the Time of Initiation of Androgen Deprivation Therapy and Time to Progression in Patients With Hormone-Sensitive Prostate Cancer. JAMA Oncol (2015) 1:495. doi: 10.1001/jamaoncol.2015.0829

12. Ventola CL. Medical Applications for 3D Printing: Current and Projected Uses. P T (2014) 39:704-11.

13. Amparore D, Pecoraro A, Checcucci E, De Cillis S, Piramide F, Volpi G, et al. 3D Imaging Technologies in Minimally-Invasive Kidney and Prostate Cancer Surgery: Which is the Urologists' Perception? Minerva Urol Nephrol (2021). doi: 10.23736/S2724-6051.21.04131-X

14. Cacciamani GE, Cuhna F, Tafuri A, Shakir A, Cocci A, Gill K, et al. Anterograde Ejaculation Preservation After Endoscopic Treatments in Patients With Bladder Outlet Obstruction: Systematic Review and PooledAnalysis of Randomized Clinical Trials. Minerva Urol Nefrol (2019) 71 (5):427-34. doi: 10.23736/S0393-2249.19.03588-4 
15. de Oliveira L, Carvalho J, Sarikaya S, Urkmez A, Salonia A, Russo GI. Patterns of Sexual Behavior and Psychological Processes in Asexual Persons: A Systematic Review. Int J Impot Res (2020). doi: 10.1038/s41443-020-0336-3

Conflict of Interest: The author declares that the research was conducted in the absence of any commercial or financial relationships that could be construed as a potential conflict of interest.
Copyright (c) 2021 Russo. This is an open-access article distributed under the terms of the Creative Commons Attribution License (CC BY). The use, distribution or reproduction in other forums is permitted, provided the original author(s) and the copyright owner(s) are credited and that the original publication in this journal is cited, in accordance with accepted academic practice. No use, distribution or reproduction is permitted which does not comply with these terms. 